\title{
Unified approach to growth and aging in biological, technical and biotechnical systems
}

\author{
Paolo Castorina ${ }^{2,3^{*}}$ and Philippe Blanchard ${ }^{1}$
}

\begin{abstract}
Complex systems, in many different scientific sectors, show coarse-grain properties with simple growth laws with respect to fundamental microscopic algorithms. We propose a classification scheme of growth laws which includes human aging, tumor (and/or tissue) growth, logistic and generalized logistic growth and the aging of technical devices. The proposed classification permits to evaluate the aging/failure of combined new bio-technical "manufactured products", where part of the system evolves in time according to biological-mortality laws and part according to technical device behaviors. Moreover it suggests a direct relation between the mortality leveling-off for humans and technical devices and the observed small cure probability for large tumors.
\end{abstract}

Keywords: Growth laws, Aging, Tumor growth

PAC Codes: $89.75 .-k$

\section{Background}

Complex systems with millions of interacting elementary parts are often considered computationally irreducible Wolfram (1984); Wolfram (2002) which means that the only way to decide about their evolution is to let them evolve in time.

On the other hand, there is an impressive number of experimental verifications, in many different scientific sectors, that coarse-grain properties of systems, with simple laws with respect to fundamental microscopic alghoritms, emerge at different levels of magnification providing important tools for explaining and predicting new phenomena.

In this respect, a priori unrelated systems show similar emergent properties and if an unexpected effect is found experimentally in a field, a similar effect, "mutatis mutandis", should also be sought in similar experiments in other fields. Therefore a useful tool to greatly facilitate the cross fertilization among different fields of research is a general classification of growth laws Castorina et al. (2006).

A very important example is the Gompertz law (GL) Gompertz (1825) which applies to human mortality tables

\footnotetext{
${ }^{*}$ Correspondence: paolo.castorina@ct.infn.it

2 Dipartimento di Fisica, Universita' di Catania, Via Santa Sofia 64, 95100

Catania, Italy

${ }^{3}$ INFN, Sezione di Catania, 95100 Catania, Italy

Full list of author information is available at the end of the article
}

(i.e. aging) and tumor growth Steel (1977); Wheldon (1988); Norton (1988).

In general, a growth problem is characterized by a function $f(t)$, which describes the time evolution of some macroscopic quantity, and by the specific rate , $\alpha$, defined as $(1 / f)(d f / d t)=\alpha(t)$. In the GL $\alpha$ has an exponential dependence on time:

$$
(1 / f)(d f / d t)=\alpha(t)=a e^{b t},
$$

where $a$ and $b$ are constants. In aging $f(t)$ indicates the survival probability; while with regards to tumor growth it corresponds to the number of cells $N(t)$ (depending on the specific case $a$ and $b$ can be positive or negative).

For technical devices the specific rate of the survival probability has a power-law time behavior

$$
(1 / f)(d f / d t)=\alpha(t)=a t^{n},
$$

with $n>1$, called Weibull law (WL) Barlow and Proschan (1975); Rigdon and Basu (2000). The analogy with the biological systems is intriguing (for clarity, as necessary, one defines the specific rate $\alpha_{h}(t)$ for the human mortality, $\alpha_{f}(t)$ for the technical systems and $\alpha_{c}(t)$ for tumor growth) and deeper than the similarity between eq. (1) and eq. (2).

Indeed, many independent analyses of experimental data on humans and animals suggest that at advanced ages (more than 85-90 years for humans) there is a

\section{是 Springer}


deceleration in mortality Gavrilov and Gavrilova (1991); Vaupel et al. (1998); Olshansky (1998): in the large range 20 - 85 years for humans the mortality rate is well described by the Gompertz law and then there is a latelife mortality (although a definite conclusion has yet to be reached Gavrilov and Gavrilova (2011)). A similar trend is observed for technical devices Economos (1979), confirming the analogy between biological and technical systems.

The understanding of aging and of late-life mortality is still an open problem and many interesting models have been proposed to explain the similar behavior in metabolic systems and in technical devices Gavrilov and Gavrilova (2001). Moreover, a unifying language for the description of performance of metabolic and technical production and distribution has been recently suggested Becker et al. (2011) to implement the idea that the robustness of metabolic systems with respect to enviromental changes could represent a useful model for technical systems.

In this letter, rather than focusing on specific models, we shall address the generalization of the classification scheme of growth laws to include human aging, tumor (and/or tissue) growth, logistic and generalized logistic growth and the aging of technical devices. We shall consider two applications of the proposed approach: a) a method to evaluate the aging/failure of combined new bio-technical "manufactured product", where part of the system evolves in time according to biological-mortality laws and part is a technical device; b) an interpretation of the "tumor size effect", i.e. the small cure probability for large tumor Stanley et al. (1977); Bentzen and Thomas (1996); Huchet et al. (2003), in analogy with the late-life mortality in aging.

\section{Results}

Let us start with the general classification scheme. It turns out that a classification of the growth laws according to the simple equation $(1 / f)(d f / d t)=\alpha(t)$ is obtained by considering the power expansion in $\alpha$ of the function (see ref. Castorina et al. (2006) for details)

$$
\Phi(\alpha)=\frac{d \alpha}{d t}=\Sigma_{i} b_{i} \alpha^{i} i=0,1,2 \ldots
$$

which for $b_{0}=0$ and $b_{i}=0$ for $i>1$ gives a time independent specific rate $\alpha_{0}$ and therefore an exponential growth; for $b_{0} \neq 0$ and $b_{i}=0$ for $i>1$ describes a linear time dependent specific rate and again an exponential growth; at the first order in $\alpha$, for $b_{0}=0, b_{1} \neq 0$ and $b_{i}=0$ for $i>1$, reproduces an exponential time behavior of the specific growth and therefore the GL; the second order term, $O\left(\alpha^{2}\right)$, for $b_{0}=0, b_{1}, b_{2} \neq 0$ and $b_{i}=0$ for $i>2$ generates the logistic and generalized logistic growth.
The feedback effect, that is the dependence of the specific growth rate $\alpha$ on the function $f(t)$, can be easily derived by the temporal behaviour of the specific rate. For the GL for a growing number of cells, $N(t)$, one has the well known logarithmic non linearity,

$$
\frac{1}{N(t)} \frac{d N(t)}{d t}=a-b \ln \frac{N(t)}{N_{0}}=b \ln \frac{N_{\infty}}{N(t)} \quad \text { Gompertz, }
$$

and for the (generalized) logistic law one gets the typical power-law behavior

$$
\frac{1}{N(t)} \frac{d N(t)}{d t}=c\left[1-\left(\frac{N(t)}{N_{\infty}}\right)^{\gamma}\right] \text { gen. logistic, }
$$

where $a, b, c, \gamma$ are constants and the carrying capacity, $N_{\infty}$,corresponds to $\alpha=0$.

In order to describe technical devices, the previous classification scheme has to be generalized since the specific growth rate of Weibull law has a power law dependence on time which is not reproduced by eq. (3). The behavior $\alpha_{f}(t) \simeq t^{n}$,with $n$ positive integer, corresponds to terms $\mathrm{O}\left(\alpha^{(n-1) / n}\right)$ in the expansion of $\Phi(\alpha)$ and therefore for a general classification scheme of the specific growth/aging/failure rate of biological and technical systems one has to consider:

$$
\Phi(\alpha)=\Sigma_{n>2}^{\infty} c_{n} \alpha^{(n-1) / n}+\Sigma_{n \geq 1} b_{n} \alpha^{n}
$$

Note that: a) $0<(n-1) / n<1$ and the nth term in the power series in $\alpha^{(n-1) / n}$ tends for large $n$ to $\alpha$, i.e. to the Gompertz law; b) the term $b_{0} \neq 0$, i.e. the exponential growth, has been neglected because one considers the GL, the generalized logistic or more complex growth laws for the biological systems (there is no problem to include this term in the expansion) ; 3 ) the first sum in the expansion has fractional powers that recall a Puiseux expansion.

As a by-product of the proposed classification scheme one can easily evaluate the aging/failure of combined new bio-technical "manufactured products" by taking explicitely into account the mutual "interference" between the aging behavior of the biological part and the failure of the technical one. The "interference" effect strongly depends on the typical time scales in the coefficients $c_{n}$ and $b_{n}$ in the previous expansion: if the life-time of the technical device is much larger than the life-time of the biological part ( or viceversa) there is essentially no effect Muller et al. (1988).

Let us first consider aging/failure of a combined biotechnological "manufactured product", where part of the system evolves in time according to GL, i.e. the term $O(\alpha)$, and the behavior of technical part is described by a single term $O\left(\alpha^{n-1 / n}\right)$,i.e.

$$
\Phi(\alpha)=c_{n} \alpha^{(n-1) / n}+b_{1} \alpha
$$


By introducing dimensionless variables in time unit $1 / b_{1}$, i.e. $\tau=b_{1} t, \bar{\alpha}=\alpha / b_{1}$ and $\bar{c}_{n}=c_{n} b_{1}^{-1-1 / n}$, after simple calculations the time dependence of the specific rate is given by:

$$
e^{\tau}=\frac{\bar{\alpha}}{\bar{\alpha}_{0}} \frac{\left[1+\left(\bar{c}_{n}\right) \bar{\alpha}^{-1 / n}\right]^{n}}{\left[1+\left(\bar{c}_{n}\right) \bar{\alpha}_{0}^{-1 / n}\right]^{n}}
$$

where $\bar{\alpha}_{0}=\bar{\alpha}(\tau=0)$. Of course in the limit $c_{n} \rightarrow 0$ one recovers the GL and for $b_{1} \rightarrow 0$ the Weibull one. By previous equation, for $\bar{\alpha}_{0}=1$, one obtains:

$$
\ln \bar{\alpha}=n \ln \left[\left(1+\bar{c}_{n}\right) e^{\tau / n}-\bar{c}_{n}\right]
$$

which describes the combined effect of the two growth laws. The quantitative effect is depicted in Figures $(1,2)$ where the previous function is plotted for different values of $n$ at fixed $\bar{c}_{n}$ and for various values of $\bar{c}_{n}$ at fixed $n$.

The next step is to include the term $b_{2} \alpha^{2}$ in the expansion of $\Phi(\alpha)$ ( $b_{2}$ is dimensionless) which corresponds to a generalized logistic evolution. As we shall see this term is crucial in understanding the late-life mortality effect.

By repeating analogous calculations it turns out that

$$
\tau=\ln \left(\bar{\alpha} / \bar{\alpha}_{0}\right)-\int_{\bar{\alpha}_{0}}^{\bar{\alpha}} d x \frac{b_{2}+\bar{c}_{n} x^{-(1-n) / n}}{1+b_{2} x+\bar{c}_{n} x^{-1 / n}}
$$

In Figure 3 is shown that the term $b_{2} \alpha^{2}$ completely changes the time evolution with respect to GL and/or WL producing a leveling-off of the specific rate.

Therefore the general expansion of $\Phi(\alpha)$ in eq. (6) can describe the aging/failure of any biological and technical

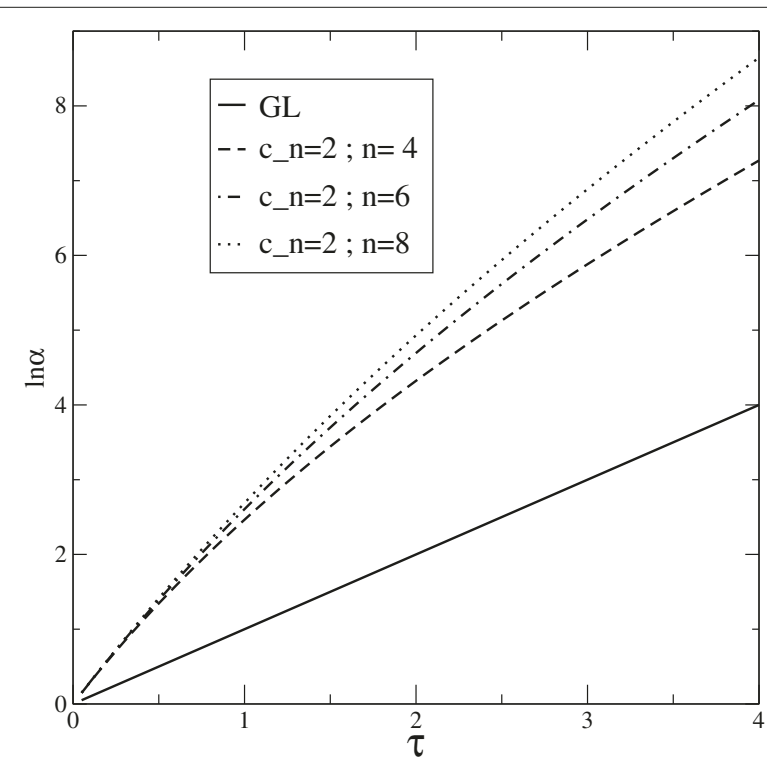

Figure 1 Comparison of the GL, the $\mathrm{WL}$ and the combined effect for a biotechnical device for $\ln \overline{\boldsymbol{\alpha}} \cdot \tau=b_{1} t$ and the curves are for a fixed value of the coefficient $\bar{c}_{n}=2$ and different values of $n=4,6,8$. system including the leveling-off at late mortality which is obtained by taking into account the term $O\left(\alpha^{2}\right)$ in $\Phi(\alpha)$, i.e. by the transition from the GL or WL to a logistic type law Horiuchi and Wilmoth (1998).

The proposed unification scheme suggests a practical method to understand growth patterns. Given a set of data on some growth process, the first step of the analysis is a fit in power of $\alpha$ of the derivative of the specific growth rate, i.e. of the function $\Phi(\alpha)$. Therefore : a) if the best fit is linear, the growth is a Gompertzian one; $b$ ) if the best fit is quadratic, look at the sign of the coefficients of the expansion. For $b_{1}>0$ and $b_{2}<0$ the growth is logistic (or generalized logistic) corresponding to a competitive dynamics; c) if the best fit indicates a fractional power the growth follows the WL. Of course, it is always possible to obtain a better agreement with data by increasing the number of coefficients. However, should increasing the number of parameters indicate only a marginal improvement in the description of data one concludes that the added terms in the expansion are irrelevant.

\section{Discussion and conclusions}

Let us now consider the cross-fertilization among different sectors.

As previously discussed, there is a deceleration of mortality in aging at late time which is described as a "transition" from a Gompertz law to a generalized logistic behavior. On the other hand, tumors evolve in time according to the GL. The obvious indications is to verify if a phenomenon corresponding to the deceleration of mortality,

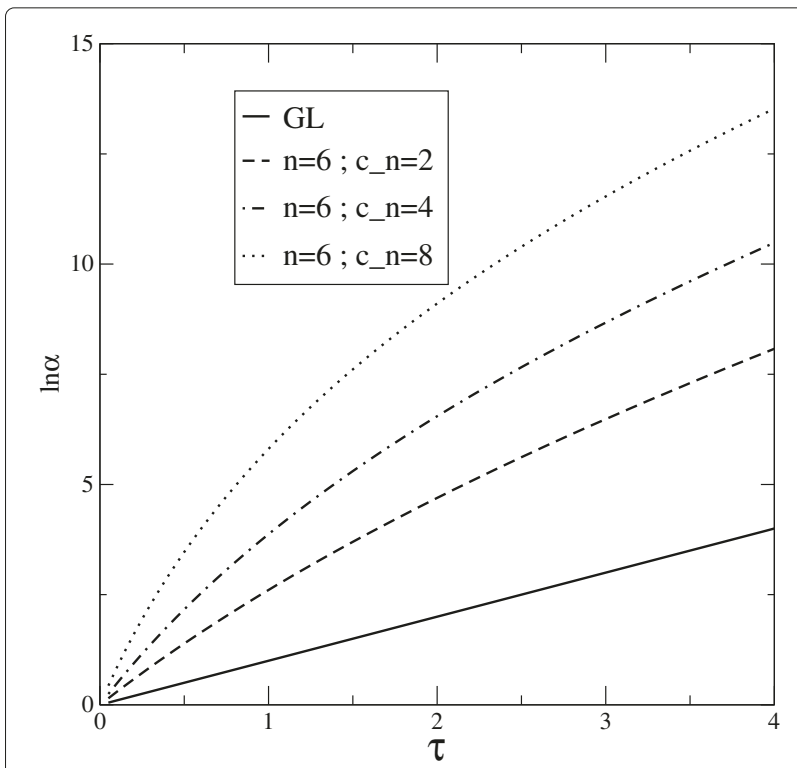

Figure 2 Comparison of the GL, the $\mathrm{WL}$ and the combined effect for a biotechnical device for $\ln \overline{\boldsymbol{\alpha}} \cdot \tau=b_{1} t$ and the curves are for $n=6$ and the coefficient $\bar{c}_{n}=2,4,8$. 


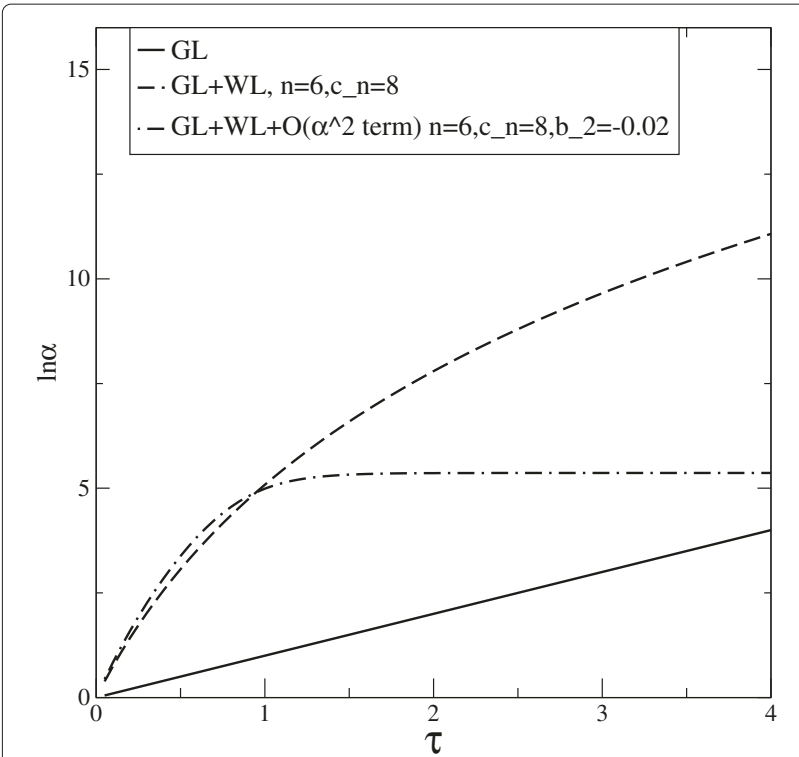

Figure 3 Comparison for $\ln \bar{\alpha}$ of the GL, the WL and the effects of $O\left(\alpha^{2}\right)$ term for $n=6, \bar{c}_{n=8}$ and $b_{2}=-0.02$.

i.e. a transition from the GL to a power law, exists for cancer growth at a later time. As we shall see, this aspect has strong consequences on the therapy.

For tumor growth the $b_{1} \alpha$ term gives the GL in eq. (4) and the introduction of the $O\left(\alpha^{2}\right)$ term corresponds to the power law non-linear feedback in eq. (5). Therefore one has to investigate if at late-life of a tumor growth there is such a modification in the dependence of the specific growth rate on the cell number $N(t)$. Since direct informations "in vivo" are almost impossible, the question has to be addressed in an indirect way by considering radiotherapy.

The radiotherapic tumor treatment consists in series of radiation doses at fixed time intervals. However tumors start to re-grow in the interval between two treatments : the re-growth during radiotherapy is therefore an important clinical parameter Kim and Tannock (2005) and the probability of treatment benefit critically depends on the tumor re-growth pattern.

The so called "tumor size effect" is a reduction of radiotherapeutic results for large tumors (which , presumably, has grown since long time). The dependence of the surviving fraction on the tumor volume was already observed by Stanley et al. in 1977 in lung tumors Stanley et al. (1977) and re-emphasized by Bentzen et al. and Huchet et al. in Bentzen and Thomas (1996); Huchet et al. (2003).

The effect of re-growth rate on radiotherapy has been quantitatively investigated in ref. Castorina et al. (2007) and the results clearly indicate that to understand the tumor size effect the re-growth rate for large tumor has to follow a power law Guiot et al. (2003) rather than the GL.
From this point of view the "tumor size effect" is a phenomenon which indicates that in late -time tumor growth there is a change from a GL specific rate to a power law behavior, corresponding to the deceleration in mortality at advanced age.

One should conclude that such a common feature in aging and in failure in biological and/or technical systems should be considered as a "bifurcation" or a "phase transition" in the specific growth rate at large time from GL or WL to a logistic or generalized logistic behavior.

In closing, the general expansion of $\Phi(\alpha)$ in eq. (6) can describe the growth/aging/failure of biological and technical systems and the transition to a different ("phase") specific growth rate at late-life could be a common feature of those systems independently on the microscopic dynamics.

\section{Competing interests}

The authors declare that they have no competing interests.

\section{Acknowledgements}

It is a pleasure to thank H. Satz for helpful comments and discussions. PC thanks for the hospitality the Department of Physics of Bielefeld University.

\section{Author details}

${ }^{1}$ Fakultät für Physik, Universität Bielefeld, D-33501 Bielefeld, Germany.

${ }^{2}$ Dipartimento di Fisica, Universita' di Catania, Via Santa Sofia 64, 95100

Catania, Italy. ${ }^{3}$ INFN, Sezione di Catania, 95100 Catania, Italy.

\section{Author's contributions}

PB and PC carried out the entire study in collaboration. Both authors read and approved the final manuscript.

Received: 31 March 2012 Accepted: 9 July 2012

Published: 9 July 2012

\section{References}

Barlow, RE, Proschan F (1975) Statistical theory of reliability and testing. Probability models. New York: Wiley

Becker T, et. al. (2011) Flow control by periodic devices: a unifying language for the description of traffic, production and metabolic systems. J Stat Mech: P05004

Bentzen SM, Thomas HD (1996) Tumor volume and local control probability: clinical data and radiobiological interpretations. Int Radiat Oncol Biol Phys 36: $247-251$

Castorina P, Deisboeck TS, Gabriele P, Guiot C (2007) Growth Laws in Cancer: Implications for Radiotherapy. Rad Res 169: 349

Castorina P, Delsanto PP, Guiot C (2006) Classification Scheme for Phenomenological Universalities in Growth Problems in Physics and Other Sciences. Phys Rev Lett 188701: 96

Economos AC (1979) A non-gompertzian paradigm for mortality kinetics of metazoan animals and failure kinetics of manufactured products. AGE 2: 74-76

Gavrilov LA, Gavrilova NS (1991). The Biology of life span: a quantitative approach. N.Y: Harwood Academic Publisher

Gavrilov LA, Gavrilova NS (2001) The reliability theory of aging and longevity, J Theor Biol 213(4): 527-545

Gavrilova NS, Gavrilov LA (2011) Mortality measurement and modeling beyond age 100, Symposium Orlando, Living to 100 monograph (published on line), The Society of Actuaries, 2011

Gompertz B (1825) On the nature of the function expressive of the law of human mortality and a new mode of determining life contingencies. Phil Trans R Soc 115: 513

Guiot C, Degiorgis PG, Delsanto PP, Gabriele P, Deisboeck TS (2003) Does tumor growth follow a 'universal law? J Theor Biol 225: 147-151

Horiuchi S, Wilmoth JR (1998) Deceleration in tha age pattern of mortality at older ages. Demography 35: 391 
Huchet A, Candry H, Belkaceni Y (2003) L'effet volume en radiotherapie. Premiere parie: effect volume et tumeur. Cancer Radiotherapie 7: 79-89 Kim JJ, Tannock IF (2005) Repopultaion of cancer cells during therapy: an important cause of treatment failure. Nat Rev Cancer 5: 516-525

Muller Ch, et al. (1988) The survival rate of patient with pace-maker is essentially the same of the normal population. Eur Heart J 9: 1003

Norton LA (1988) Gompertzian model of human breast cancer growth. Cancer Res 48: 7067-7071

Olshansky S J (1998) On the biodemography of aging: a review essay Population and Development Review 24: 381-393

Rigdon S E, Basu A P (2000). Statistical methods for the reliability of repairable systems. New York: Wiley

Stanley J A, Shipley W U, Steel G G (1977) Influence of tumor size on the hypoxic fraction and therapeutic sensitivity of Lewis lung tumour. Br J Cancer 36: 105-13

Steel G G (1977). Growth kinetics of tumours. Oxford: Clarendon Press Vaupel J W, Carey J R, Christensen K, Johnson T, Yashin A I, Holm N V, lachine I A, Kannisto V, Khazaeli A A, Liedo P, Longo V D, Zeng Y, Manton K, Curtsinger J W (1998) Biodemographic trajectories of longevity. Science 280: 855-860

Wheldon, TE (1988). Mathematical models in cancer research. Bristol: Adam Hilger Publisher

Wolfram S (1984) Nature 311:419-424

Wolfram S (2002). A New Kind of Science. Wolfram media, Champaign, USA

doi:10.1186/2193-1801-1-7

Cite this article as: Castorina and Blanchard: Unified approach to growth and aging in biological, technical and biotechnical systems. SpringerPlus $20121: 7$

\section{Submit your manuscript to a SpringerOpen ${ }^{\circ}$ journal and benefit from:}

- Convenient online submission

- Rigorous peer review

- Immediate publication on acceptance

- Open access: articles freely available online

- High visibility within the field

- Retaining the copyright to your article 